\title{
Refraction changes in children developing convergent or divergent strabismus
}

\author{
Maths Abrahamsson, Gerhard Fabian, Johan Sjöstrand
}

\begin{abstract}
Strabismus and amblyopia were studied in a cohort of children born in 1979 or 1980 in the area of Västerås, Sweden. Forty percent of the children had participated in a voluntary eye examination at 1 year of age. All children diagnosed as strabismic and/or amblyopic between 1979 and 1988 at any of the three eye clinics in the area were included in this study. Strabismic cases were mostly detected by the parents while microstrabismus and straight eye amblyopia were found at the general 4 years of age screening at children's health centres. In 57 cases with $(n=31)$ and without amblyopia $(n=41)$ it was possible to obtain several refraction values between 1 and 6 years of age. In this study we concentrated on manifest esotropia and exotropia. The aim of the study was to describe changes of refraction before and after onset of strabismus and to establish risk indicators that identified populations at risk of developing strabismus. We found that patients with esotropia show a more pronounced hypermetropia than exotropic cases at the time of detection of strabismus. This difference becomes more definite over time, since hypermetropia increased in the deviating eye in the esotropic cases while refractive errors remained stationary in most of the exotropic eyes. It was also apparent that anisometropia frequently developed after onset of strabismus in esotropic cases in contrast to exotropic cases. An increasing refractive error in the deviating esotropic eye could be combined with an emmetropisation of the fixating eye. (Br f Ophthalmol 1992; 76: 723-727)
\end{abstract}

During the past two decades there has been a focus of interest on the changes of refraction during infancy and childhood. Previously it was believed that infants were hypermetropic at birth and that there was a decrease in hypermetropia during infancy and childhood. Later it was determined that as many as $20 \%$ of the newborn are myopic. ${ }^{12}$ Other studies have shown that after the infantile period (at 1 or 2 years of age) most children are hypermetropic. ${ }^{13}$ From the age of approximately 1 year there is a decrease in refractive errors, especially in the amount of astigmatism. ${ }^{3-5}$ These data together with other studies indicate that there are considerable changes in refraction during the first years of life. . $^{6-8}$

One reason for this growing interest is the possibility of using refractive errors as risk indicators useful in screening programmes for young children in order to prevent amblyopia and squint. Knowledge of potentially dangerous refractive anomalies is necessary in order to define the population at risk. The high variability of the individual refraction during early childhood complicates the determination of risk indicators. Age becomes an essential factor for evaluation of when a refraction error is potentially detrimental for visual development.

In this study we were interested in the refractive changes of children who develop strabismus. In patients with convergent strabismus we have known that there is a relationship to hypermetropia since 1864 when Donders presented his publication 'On the anomalies of accommodation and refraction of the eye.' This has later been confirmed by several studies (cf Duke Elder). " The longitudinal changes of refraction in esotropic individuals have also been studied, ${ }^{10-12}$ and one study has examined refractive changes before the onset of strabismus. " Though these studies ${ }^{9-12}$ all found a high hyperopia in most cases with esotropia it is still difficult to determine a refraction limit at any particular age that separates potentially esotropic children from normal ones.

In divergent strabismus, early studies by Donders indicated an association with myopia. This relationship was questioned however at the beginning of this century. ${ }^{9}$ There seems to be a portion of myopia among the exotropic children but the majority are hypermetropic at onset.

The aim of this study was to compare refraction changes in children with manifest esotropia or exotropia compared with the development of the refractive errors in normal children. We also wanted to examine refractive changes before and after the onset of the strabismus to detect whether abnormal refractive behaviour could be used as an indicator for pointing out patients at risk of developing strabismus and/or amblyopia.

\section{Methods}

\section{SUBJECTS}

Refraction data for the study were obtained by combining two different studies. In the first we examined, motility, fixation, refractive errors, and heredity of 9000 children at 1 year of age. These children took part in a voluntary ophthalmic examination (1971-81). The examination was offered through children's health care centres to all children in the city of Västerås, Sweden. Approximately $50 \%$ of the children born in the area took part in the examination.

In the second study we scrutinised the hospital files of all children born between 1979 and 1980 who visited one of the three ophthalmic clinics in the city of Västerås, Sweden, during the 1980s. We found 715 cases that for some reason visited an eye clinic before 8 years of age. There were a 
Table 1 Distribution of strabismus

\begin{tabular}{llllllc}
\hline & Esotropia & Exotropia & Microtropia & Infantile & Paralytic & Total \\
\hline $\begin{array}{l}\text { Number of patients } \\
\text { Percentage of strabismic }\end{array}$ & 41 & 21 & 8 & 6 & 4 & 80 \\
$\begin{array}{c}\text { patients } \\
\begin{array}{c}\text { Percentage of all children } \\
\text { born in the area 79, 80 }\end{array}\end{array}$ & 1.4 & 26 & 10 & 8 & 5 & 100 \\
\hline
\end{tabular}

large variety of reasons for the visits. Most common were conjunctivitis, trauma, strabismus, or suspected amblyopia. In 290 of these patients we have refraction data from the voluntary examination at 1 year of age that could be analysed together with data from the first visit to an eye clinic. In 57 cases with strabismus $(n=31)$ and/or amblyopia $(n=41)$ it was possible to obtain a series of refraction values between 1 year of age and up to 5 or 6 years of age.

The patients were refracted by retinoscopy 30 minutes after instillation of cyclopentolate (1\%). Spectacles were prescribed at detection of the strabismus in patients with hypermetropia $\geq 2 \mathrm{D}$. The anisometropia of the strabismic and amblyopic patients was fully corrected. None of the amblyopic and/or strabismic cases wore spectacles before detection of strabismus/ amblyopia. Time of detection was defined as the date when strabismus is diagnosed by an ophthalmologist or by an orthoptist.

In this study we describe refraction and refraction changes and their significance in patients with manifest strabismus. The reliability of the data depends on the accuracy of the refraction data and ability of the screening programme to detect patients with amblyopia and strabismus and refer them to an eye clinic. In our earlier studies all refraction determinations have been done by one ophthalmologist (GF) with much experience of refraction determination in young children. ${ }^{131314}$ All refraction measurements with young children were performed by GF while some refraction data in older children were obtained from several other ophthalmologists at different eye clinics. We are aware of the problem of having several ophthalmologists involved and have tried to minimise the effects.

We have chosen to follow longitudinal changes in refraction from 1 to 4 years in the majority of cases. The reason for choosing 4 years as an endpoint is that we have a general health screening in Sweden at the age of 4 years. More than $95 \%$ of all children take part in that screening. Studies indicate that most amblyopic

Figure 1 The age at which amblyopia is diagnosed by an ophthalmologist at an eye clinic in the city of Västeras. Sweden, is presented for all children born in 1979 or children born in 1979 or 1980. The hatched columns indicate those children with amblyopia identified by the screening programme at approximately 4 years of age.

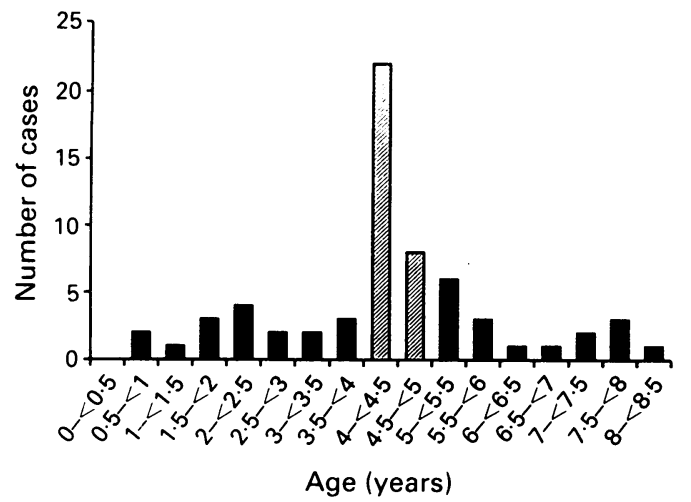

cases that are not previously known are identified at this screening. ${ }^{516}$

STRABISMUS AND AMBLYOPIA

The cases of manifest strabismus $(n=80)$ were classified into one of five categories; esotropia, exotropia, microstrabismus, infantile, and paralytic strabismus (Table 1). Infantile strabismus was defined as a manifest strabismus detected before 9 months of age. All patients with predominantly intermittent strabismus were excluded from the study. We concentrated especially on the cases with esotropia and exotropia. The cases with infantile esotropia and microstrabismus were not included in the comparison of refraction changes (Figures 4 and 5).

Straight eye amblyopia and microstrabismus are generally detected by the Swedish general health screening ${ }^{15}$ and cases with strabismus are referred to an eye clinic by the children's health care centres. Children with monocular visual acuity lower than 0.8 or an interocular difference in acuity of two rows or more (HVOT or tumbling E letter chart) at the 4 year screening are retested within the screening programme. Children with subnormal vision at both these test sessions are referred to an eye clinic. We have chosen to use a somewhat uncommon definition of amblyopia in our analysis of the hospital files. A patient must fulfil the following two criteria in order to be classified as amblyopic: (1) an acuity difference (approximately $0 \cdot 1 \mathrm{log}$ unit or more, which equals 1 line or more) between the eyes measured at the eye clinic with best correction at least two separate consecutive test sessions in eyes with no signs of disease; (2) the acuity of the 'amblyopic' eye improved at least one line following the occlusion treatment.

The first criterion means that a child will have his visual acuity tested four times before he or she is classified as amblyopic if referred from the general health screening programme. Children referred from sources outside the screening programme pass two visual acuity tests or more before they are classified as amblyopic. The amblyopic cases $(n=126)$ were classified into

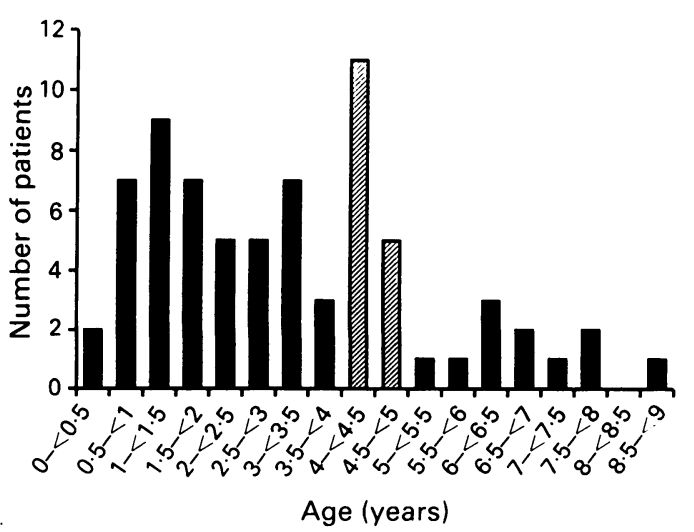

Figure 2 The age at which strabismus is diagnosed by an ophthalmologist is presented for all children born in 1979 or 1980 and visiting an eye clinic in the city of Västeras, Sweden. In the diagram both manifest and intermittent strabismus are included. The hatched columns indicate those children with strabismus (mainly microstrabismus) identified by the screening programme at approximately 4 years of age. 
Figure 3 The relative distribution of the age at detection of cases with manifest strabismus is presented for children with esotropia and exotropia respectively. The column 0-1 year contains only cases detected between 9 and 12 months of age.

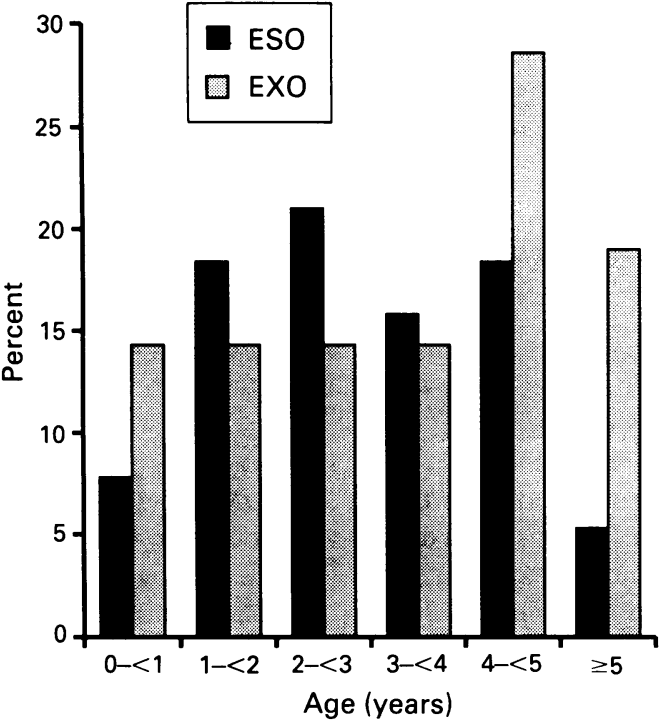

three different groups according to the severity of the visual loss. The best corrected visual acuity (VA) of the amblyopic eye at the start of treatment was graded in three levels as follows: $\mathrm{VA} \leq 0 \cdot 2,0 \cdot 2<\mathrm{VA} \leq 0 \cdot 5$, and $\mathrm{VA}>0 \cdot 5$.

\section{Results}

In Swedish children there are different patterns for detection of strabismus compared with amblyopia. Cases with amblyopia without eye deviation or with microstrabismus are mostly identified by the visual screening programme. All $(>95 \%)$ of the Swedish children have a general health examination, including an eye examination, at 4 years of age. In Figure 1 the age at detection of amblyopia is presented. The cases identified earlier than 4 years of age usually have large angle strabismus or amblyopia caused by visual deprivation (that is, infantile cataract, ptosis). The detection pattern of strabismus is different (Fig 2). The age of detection is evenly distributed between 1 and 4 years. The screening programme identifies undetected microstrabismic cases while cases with manifest large angle strabismus are identified by the parents. These cases are referred by the children's health centre to an ophthalmologist shortly after onset of the strabismus. In Table 1 the distribution of the different types of manifest strabismus is presented both as numbers and as a percentage of

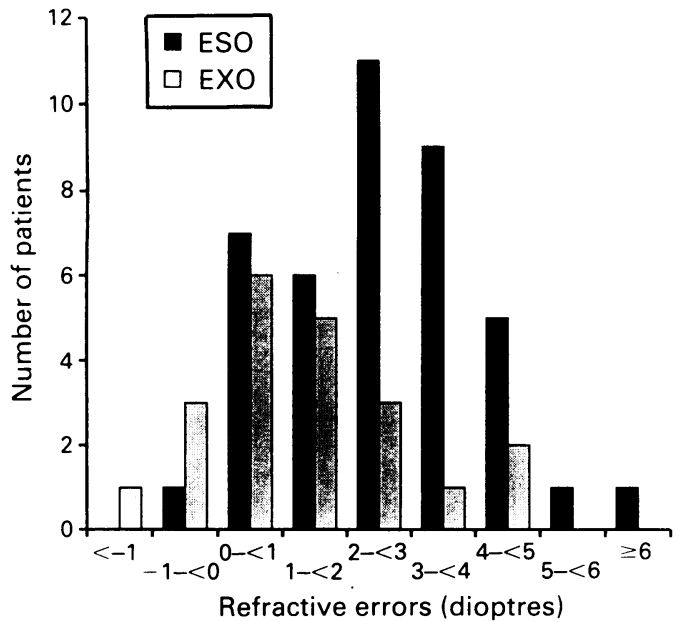

patients found in hospital files, and as a percentage of all children born in the area. Approximately $75 \%$ of all cases with strabismus were classified as exotropia or esotropia. Infantile, paralytic, and microstrabismus represented approximately one quarter of all strabismus diagnoses. The ratio between number of cases with convergent and divergent strabismus was approximately $2: 1$.

REFRACTION AND REFRACTION CHANGES

For the study of refraction we have concentrated our analysis of the refraction changes with time in cases with manifest esotropia and exotropia $(n=62$, Table 1). No cases of infantile strabismus (detected earlier than 9 months of age), paralytic strabismus, microstrabismus, or predominantly intermittent strabismus were included. The age at detection of the two types of strabismus is presented in Figure 3. The onset of esotropia and exotropia is distributed differently. Most $(\approx 95 \%)$ esotropic cases are detected between 1 and 4 years of age while more than $40 \%$ of the exotropic cases are detected later than 4 years of age.

At the time of detection of strabismus, astigmatism ( $\geq 1.0 \mathrm{D})$ is common $(29 \%$ of all cases) in both eyes in both convergent and divergent strabismus while anisometropia is more uncommon in both esotropia and exotropia (esotropia $n=5 / 41$; exotropia $n=3 / 21$ ). The distribution of the refractive error (spherical equivalent) in the deviated eye at detection, however, is different between the two groups of strabismus (Fig 4). More than $60 \%$ of the esotropic patients have a hypermetropia that is more than 2 dioptres in the deviating eye at the date of detection while we found no case with myopia in this group. It is notable that in seven $(18 \%)$ of the esotropic cases the refractive error was less than 1 dioptre in any meridian. In exotropia larger refraction errors are more

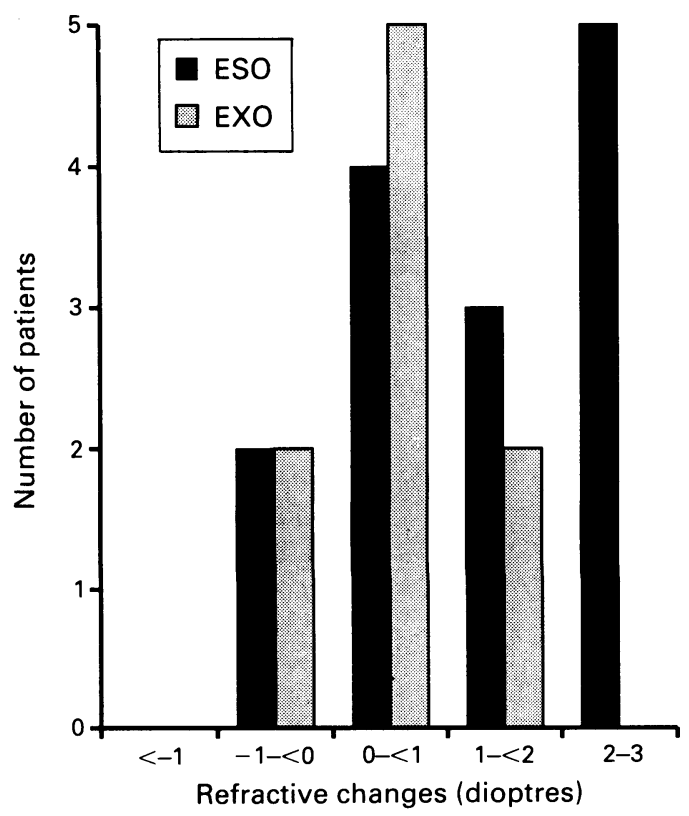

Figure 5 The longitudinal changes in refraction of the deviating eye of children with manifest esotropia and exotropia respectively. The refraction changes are calculated as spherical equivalent at 4 years of age minus spherical equivalent at 1 year of age. 
Table 2 Distribution of amblyopia

\begin{tabular}{lllll}
\hline & $V A \leq 0.2$ & $0.2<V A \leq 0.5$ & $0.5<V A$ & Total \\
\hline $\begin{array}{l}\text { Number of patients } \\
\text { Percentage of amblyopic }\end{array}$ & 11 & 46 & 69 & 126 \\
$\begin{array}{c}\text { patients } \\
\text { Percentage of all children }\end{array}$ & 8.7 & 36.5 & 54.8 & 100 \\
\begin{tabular}{l} 
born in the area 79,80 \\
\hline
\end{tabular} & 0.3 & 1.5 & 2.3 & 4.1 \\
\hline
\end{tabular}

uncommon. In $65 \%$ of the cases the errors are between -1 and $+2 \mathrm{D}$ in the deviating eye. One exotropic patient has a pronounced myopia $(-8 \mathrm{D})$ in both eyes.

In order to study differences of refractive errors in the deviating eye at onset two age groups were compared: children younger than 36 months at onset (but older than 9 months) and older than 36 months. No significant difference in the distribution of refractive error in convergent or divergent squint respectively could be found for the two age groups. The same differences between exotropia and esotropia as described above were thus found in both age groups.

The refractive changes between 1 and 4 years of age could be studied in 23 cases with nonalternating strabismus, 14 esotropic (14/41), and nine exotropic (9/21) patients (Fig 5). At 1 year of age one esotropic and two exotropic patients are myopic and five esotropes and three exotropes had a hypermetropia greater or equal to $3.5 \mathrm{D}$ in the most hypermetropic meridian in any eye. The majority of the esotropic patients (10/14) show an increase in hypermetropia $\geq 0.5 \mathrm{D}$ in the strabismic eye during the period from 1 to 4 years. Increasing hyperopia could be identified both before and after the detection of the esotropia in more than $50 \%$ of the deviating eyes while in several cases there was an emmetropisation or at least a decreasing hypermetropia in the fixating eye. In the esotropic patients anisometropia developed owing to this difference in behaviour between the eyes. At 1 year of age as well as at onset of the esotropia there is one (1/14) case with anisometropia. One year after onset (11-16 months) 13 of the esotropes had developed anisometropia greater or equal to $1 \mathrm{D}$. Most patients with divergent squint showed small $(\leq 0.5 \mathrm{D})$ or no changes in the refraction in either eye (7/9). The same process of developing anisometropia as in convergent squint could not be seen.

STRABISMUS AND AMBLYOPIA

The distribution of acuity of all amblyopic eyes is presented in Table 2 . We used the amblyopia definition presented in the Methods section and classified the patients into different groups according to the severity of the visual loss. In more than $50 \%$ of the cases with amblyopia the visual acuity in the amblyopic eye was better than 0.5 . When we combine the distributions of amblyopia and strabismus (Tables 1 and 2) in the whole population we found that in our estimation of distribution $2 \cdot 7 \%$ of all children had strabismus and $4.2 \%$ had amblyopia, while the percentage with both amblyopia and strabismus was $1 \cdot 4 \%$.

Amblyopia was present in $53 \%$ of all the cases with manifest strabismus $(42 / 80)$ while patients with squint represented $33 \%$ of all amblyopic cases $(42 / 126)$. When we study only severe amblyopia (VA $<0.2$ ) the relationship between amblyopia and strabismus is much more pronounced: $72 \%$ of the patients with severe amblyopia were also squinting (8/11). The distribution of convergent and divergent strabismus into different amblyopia categories was very similar. Approximately $20 \%$ in both groups of strabismus developed severe amblyopia (VA < $0 \cdot 2)$. The same was true for microstrabismus while all cases of paralytic strabismus presented with amblyopia of a low magnitude (VA $>0.5$ ). The number of patients is small so these data can suffer from random errors.

The treatment for amblyopia was unsuccessful in six cases out of $80(7 \cdot 5 \%)$ with strabismus. Unsuccessful means a difference in acuity of two rows or more after the conclusion of the occlusion treatment. Five of the cases who remained amblyopic despite treatment had convergent squint, and in only one case was a divergent squint present. That child also had nystagmus. Among the five children with esotropia and amblyopia persisting after treatment two cases had severe amblyopia.

\section{Discussion}

There is a limited knowledge of the causes of manifest squint. However, in some cases the general mechanisms that produce the squint are known. Brain damage, neuromuscular anomalies, and abnormalities of the fusion mechanism are related to motor problems that can cause squint. Strabismus can also be caused by mechanical factors in the orbit. Finally there is an established relationship between accommodation, refraction, and concomitant squint. Several studies have pointed out that hypermetropia and anisometropia are overrepresented in cases with convergent squint. "12 In our study we found that almost two thirds of the esotropes had hypermetropia (spherical equivalent) - that is, over $2 \mathrm{D}$ in the deviating eye - whereas only $7 \%$ had anisometropia at onset. The relationship between hyperopia and esotropia is most probably causal in many of the cases. There is a plausible aetiological connection through accommodation and the strabismus may be cured by correcting the refraction error.

The relationship between ametropia and the development of strabismus is discussed in most textbooks. Both anisometropia and hypermetropia, as well as pronounced astigmatism, have been said to trigger the development of strabismus owing to disturbances of fusion. More recent studies ${ }^{17}$ have pointed out meridional hypermetropia of +3.5 or more in a child at 1 year of age as a useful risk indicator both for amblyopia and strabismus. In our study we found that five of 14 cases with esotropia had a hypermetropia of +3.5 or more at 1 year of age. This number has increased to seven of 14 esotropes at onset of the strabismus. The connection between age and refractive error has proved to be ever more interesting, since there is a high variability and change in refraction during the first years of life. ${ }^{13+5 \times 1 \times}$ In earlier studies we 
found that in certain cases more than 4 dioptres of astigmatism and anisometropia may disappear during the period 1 of 4 years. ${ }^{31314}$

In this study we found that most of the children before onset of their convergent squint had a hypermetropia of $>2 \mathrm{D}$ or more in the deviating eye at 1 year of age while it was uncommon with hypermetropia $\geq 2 \mathrm{D}$ at the same age in the deviating eye in cases with future divergent squint. In a normal control eye the refractive error should have decreased to $1-1.5 \mathrm{D}$ at the age of 4 years. Studying the refractive error at 4 years we found a very limited decrease in refraction of the deviating eye of both groups (Fig 5). Among the convergent squinters there was even an increase in the refractive error in most cases, with as much as $2.75 \mathrm{D}$ in one case. If esotropia was characterised by an increasing refractive error divergent squint was characterised by a lack of emmetropisation of the deviating eye. In most of the exotropic cases there was no change in refraction at all during the test period of 1 to 4 years.

A most interesting finding was that some esotropic cases developed anisometropia after the onset of the strabismus. Seven percent of the esotropes had anisometropia at onset which could be compared with the $10 \%$ we found for the whole population at that age level ${ }^{13}$ (and unpublished data). Among the 14 esotropic cases which could be followed with respect to refraction between 1 and 4 years one case had anisometropia at onset of strabismus. At 1 year after (11-16 months) onset 13 of 14 children had developed anisometropia. This tendency to develop anisometropia following onset in esotropic patients can also be seen in other studies of longitudinal changes in refraction in the deviating eye before and after onset of strabismus. ${ }^{111}$ In our study anisometropia was found in all but one case of convergent strabismus at 1 year after the onset of the squint. Detailed study of the refractive changes indicates that the anisometropia is created owing to the fact that the fixating eye is emmetropising, while this process is interrupted in the deviating eye. We even found increasing refractive errors in the deviating eye. Data from Aurell and Norrsell" indicate disturbances in the emmetropisation process in both eyes before the onset of the esotropia in children with a family history of strabismus. Since our data are collected without a specific time schedule for the testing it is impossible to calculate any mean difference in time between the onset of strabismus and the onset of anisometropia. Among the patients with divergent squint no case could be demonstrated with anisometropia developing after the onset of the squint.

In a study of the relationship between refraction and amblyopia ${ }^{14}$ we found that an increasing refractive error between 1 and 4 years of age was related to the development of amblyopia in cases without deviation. The same seems to be true for the development of convergent strabismus. In this study we found that no child developed esotropia which had a decreasing refraction in the deviating eye before onset. Development of esotropia without aniso- metropia at onset was the most common finding. After onset we found cases with anisometropia and esotropia without amblyopia but no case with amblyopia and esotropia without anisometropia among those cases longitudinally followed. It is known that one can have anisometropia and amblyopia without esotropia. From those findings one may conclude that both esotropia and amblyopia are strongly associated with anisometropia; the combination of these particular conditions has the potential to cause anisometropia.

In divergent squint refraction seems to be neither a cause nor a risk indicator. We found no relationship between refraction and refraction changes and the development of divergent strabismus.

In conclusion, there is evidence that convergent and divergent squint are caused by different mechanisms. Refraction changes between 1 and 4 years of age characterised by failure of emmetropisation are observed both in cases developing convergent strabismus or amblyopia without any deviation. Anisometropia is mainly a secondary phenomenon in convergent squint while it can be a primary condition in divergent squint.

This study was supported by Swedish Medical Research Council (grant 02226), Handlanden $H$ Svensson foundation, the DBV association, and the first of May flower annual campaign for
children's health. We are grateful to the staff at the Eye Clinic at Centrallasarettet, Herrgärdets Vårdscentral and Västra Sjukhuset in the City of Västerås. Finally, we will also thank Ann-Katrin Andersson and orthoptist Eileen Bentley for their invaluable support.

1 Fabian G, Wendell ME. Ophthalmological and orthoptic examination of 1200 children up to age 2. Am Orthopt $\mathcal{F} 1974$; 24: 86-90.

2 Goldschmidt E. Refraction of the newborn. Acta Ophthalmol (Kbh) 1969; 47: 570-8.

3 Abrahamsson M, Fabian G, Sjöstrand J. Changes in astigmatism between the ages of 1 and 4 years: a longitudinal study. Brf Ophthalmol 1986; 92: 145-9.

4 Atkinson J, Braddick O, French J. Infant astigmatism: its disappearance with age. Vision Res 1980; 20: 891-3.

5 Gwiazda J, Scheiman M, Mohindra I. Astigmatism in children: changes in the axis and amount from birth to 6 years. Invest Ophthalmol Vis Sci 1984; 25: 88-92.

6 Slapater FJ. Age norms of refraction and vision. Arch Ophthalmol 1950; 43: 466-81.

7 Hirsch MJ. Refraction in children. Am f Optom 1964; 41: 395-

8 Ingram RM, Barr A. Changes in refraction between the ages of 1 and 3.5 years. Brf Ophthalmol 1979; 63: 339-42.

9 Duke-Elder S, Wybar K. Ocular motility and strabismus. System of Ophthalmology. S Duke-Elder, ed. 15 vols. London: Henry Kimpton, 1973; VI: 577-641.

10 Lepard CW. Comparative changes in the error of refraction between fixing and amblyopic eyes during growth and development. Am $\mathcal{F}$ Ophthalmol 1975; 80: 485-90.

11 Aurell E, Norrsell K. A longitudinal study of children with a family history of strabismus: factors determining the incidence of strabismus. Br 7 Ophthalmol 1990; 74: 589-94.

12 Otsuka J, Sato Y. Supplementary study of comparison of refractive components of orthophoric hyperopic eyes with those of accommodative esotropic eyes. Acta Soc Ophthalmol those of accommoda 1984 ; $58-64$

13 Abrahamsson M, Fabian G, Sjöstrand J. A longitudinal study of a population based sample of astigmatic children. II. The changeability of anisometropia. Acta Ophthalmol (Kbh) $1990 ; 68: 435-40$

14 Abrahamsson M, Fabian G, Andersson A-K, Sjöstrand J. A longitudinal study of a population based sample of astigmatic children. I. Refraction and amblyopia. Acta Ophthalmol (Kbh) 1990; 68: 428-34.

5 Köhler L, Stigmar G. Vision screening of four-year-old children. Acta Paediatr Scand 1973; 62: 17-27.

16 Köhler L, Stigmar G. Visual disorders in 7-year-old children with and without previous vision screening. Acta Paediatr Scand 1978; 67: 373-7.

17 Ingram RM, Walker C, Wilson JM, Arnold PE, Dally S. Prediction of amblyopia and squint by means of refractive at age one year. Br $\mathcal{F}$ Ophthalmol 1986; 70: 12-5.

18 Fulton AB, Dobson Y, Salem D, Mar C, Petersen RA, Hansen RM. Cycloplegic refraction in infants and young children. Am $\mathcal{F}$ Ophthalmol 1980; 90: 239-47. 